Universidade de São Paulo

Instituto de Física

\title{
Modelos com infinitos estados absorventes analiticamente solúveis
}

\author{
Evandro Freire da Silva
}

Dissertação submetida ao Instituto de Física da Universidade de São Paulo para a obtenção do título de Mestre em Ciências.

Orientador: Prof. Dr. Mário José de Oliveira

Banca Examinadora:

Prof. Dr. Mário José de Oliveira - IF-USP

Prof. Dr. Ronald Dickman - UFMG

Profa. Dra. Vera Boholometz Henriques - IF-USP

São Paulo

2005 


\section{FICHA CATALOGRAFICA}

\section{Preparada pelo Serviço de Biblioteca e Informação}

do Instituto de Física da Universidade de São Paulo

Silva, Evandro Freire da

Modelos com Infinitos Estados Absorventes

Analiticamente Solúveis. São Paulo - 2005

Dissertação (Mestrado) - Universidade de São Paulo

Instituto de Física - Departamento de Física Geral

Orientador: Prof. Dr. Mário José de Oliveira

Área de Concentração: Física

Unitermos

1. Física da Matéria Condensada;

2. Mudança de Fase - Modelos em Reticulados;

3. Mecânica Estatística - Ensembles. 
A todas as pessoas que, um dia, já se perguntaram porque o mundo é assim, e tiveram a coragem de procurar respostas. 


\section{Agradecimentos}

À minha mãe, que, por anos, tem se sacrificado para dar a mim e a meus irmãos as melhores condições materiais, afetivas e espirituais de vida.

Ao professor Mário de Oliveira, que aceitou me orientar neste trabalho e dedicou parte de seu tempo a me auxiliar neste início de caminhada na Física.

Aos professores do Departamento de Física Geral, especialmente a Tânia Tomé, Vera Henriques e Sílvio Salinas, que me auxiliaram durante o tempo em que estive envolvido neste trabalho de diversas maneiras.

Aos colegas e funcionários deste Instituto, especialmente a Carlos, Kelly, Henrique, Gustavo, André, Alexei, Leonardo, Marcus, Átila, Evaldo, Fabiano e Marco Aurélio, pela ajuda e pelo companheirismo.

À FAPESP, pela ajuda financeira.

E, acima de tudo, a Deus, sem O qual nada disso teria sido possível. 


\section{Resumo}

Neste trabalho estudamos alguns modelos com conservação de partículas, que apresentam uma transição de fase entre um estado estacionário ativo e infinitos estados absorventes. Os estados estacionários ativos de cada modelo são compostos por configurações equiprováveis, correspondendo, de acordo com a formulação gibbsiana da Mecânica Estatística, a um ensemble microcanônico. Efetuando uma mudança de ensemble, podemos calcular as grandezas físicas para cada um destes modelos utilizando a técnica de matrizes de transferência, explicada neste trabalho. Realizamos simulações destes modelos e confirmamos as hipóteses que sustentam o uso desta técnica. Por fim, analisamos dois modelos derivados dos anteriores que não podem ser estudados com base nesta técnica. 


\begin{abstract}
In this work we studied some models with particle conservation which present a phase transition between an active stationary state and infinitely many absorbing states. The active stationary states of each model consist of equiprobable configurations, corresponding, according to Gibbs's formulation of Statistical Mechanics, to a microcanonical ensemble. Carring out an ensemble change, we can calculate the physical quantities for each one of these models using the transfer matrix technique, explained in this work. We performed simulations of these models and confirmed the hypothesis that sustain the use of this technique. Finally, we analysed two models derived from the previous ones for which this technique cannot be applied.
\end{abstract}




\section{Sumário}

1 Introdução 3

1.1 Criticalidade auto-organizada ................ 3

1.2 Modelos com infinitos estados absorventes . . . . . . . . . . . 4

2 Considerações teóricas $\quad 7$

2.1 Processos estocásticos em reticulados . . . . . . . . . . . 7

2.2 Ensembles estatísticos . . . . . . . . . . . . . . 9

3 Matriz de transferência $\quad 12$

3.1 Grandezas dependentes de três ou mais sítios consecutivos . . . . . 16

3.2 Extensão do formalismo para o caso de mais de uma grandeza con-

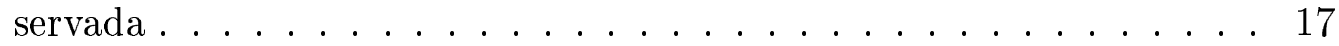

4 Modelo de aglomerados vacantes (1) 20

4.1 Apresentação do modelo (1) . . . . . . . . . . . . . 22

4.2 Caracterização do estado ativo . . . . . . . . . . . . . 24

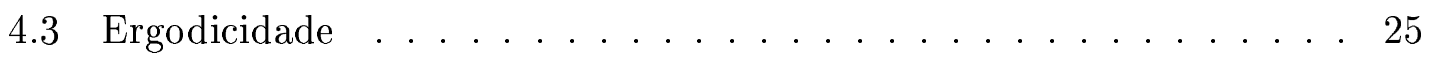

4.4 Cálculo da matriz de transferência . . . . . . . . . . . . . 26

4.5 Resultados numéricos . . . . . . . . . . . . . . . 29

5 Modelo de aglomerados vacantes (2) 32

5.1 Caracterização do estado ativo . . . . . . . . . . . . . 33

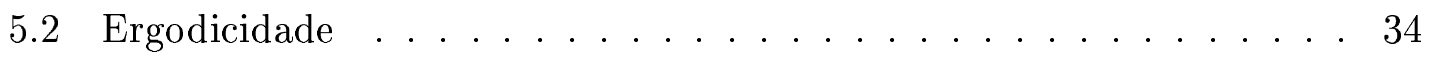

5.3 Cálculo da matriz de transferência . . . . . . . . . . . . . 35

5.4 Resultados numéricos . . . . . . . . . . . . . 38

6 Modelo de aglomerados vacantes $(1,2) c \quad 40$

6.1 Caracterização do estado ativo . . . . . . . . . . . . . 41 
6.2 Ergodicidade ........................ 45

6.3 Cálculo da matriz de transferência. . . . . . . . . . . . . . . 47

6.4 Resultados numéricos . . . . . . . . . . . . . 50

7 Modelo de aglomerados vacantes $(1,2) \quad 54$

7.1 Caracterização do estado ativo . . . . . . . . . . . . 55

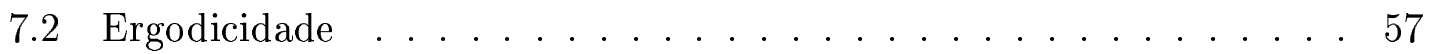

7.3 O intervalo $1 / 3 \leq \rho \leq 0.4 \ldots \ldots \ldots$. . . . . . . . 60

7.4 Cálculo da matriz de transferência . . . . . . . . . . . . . 61

7.5 Resultados numéricos . . . . . . . . . . . . . 67

8 Contra-exemplos $\quad 70$

8.1 Modelo A . . . . . . . . . . . . . . . 70

8.2 Modelo B - PCCI de segundos vizinhos . . . . . . . . . . 74

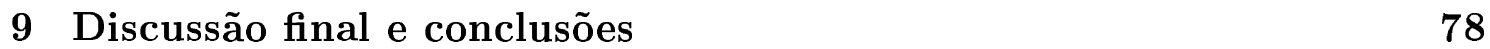




\section{Capítulo 1}

\section{Introdução}

\subsection{Criticalidade auto-organizada}

Nos útlimos anos, o conceito de criticalidade auto-organizada (CAO) tem sido utilizado para descrever uma série de modelos e sistemas físicos distintos, constituídos por muitas unidades que interagem fortemente entre si e muito mais fracamente com um ambiente externo, os quais exibem características comuns, como a obediência a leis de potências, que caracteriza uma invariância de escala temporal e/ou espacial. Podemos destacar entre os modelos principais o modelo de pilhas de areia de Bak, Tang e Wiesenfeld [1] e de Manna [2], e modelos envolvendo dinâmica extrema, como o modelo de Bak-Sneppen [3]. Dentre os sistemas físicos para os quais se postula $\mathrm{CAO}$, temos o movimento de vórtices em supercondutores observado por Field, Witt e Nori [4].

O conceito de CAO originou-se em [1], com a hipótese de que sistemas constituídos de muitas unidades interagentes pode organizar-se espontaneamente, formando uma estrutura complexa. Para que isso ocorra, as interações entre componentes do sistema devem dominar a evolução do sistema, enquanto as interações externas ocorrem muito mais lentamente, devido à existência de um limite inferior para a intensidade deste processo, abaixo do qual ele não ocorre.

O termo criticalidade ocorre comumente na termodinâmica de equilíbrio, referindo-se ao comportamento do sistema enquanto um parâmetro externo, por exemplo, a temperatura, é variado de forma que o sistema passa por uma transição de fase contínua. Quando o parâmetro do sistema é fixado no ponto onde ocorre esta transição, isto é, no ponto crítico, observa-se que uma perturbação local induzida no 
sistema propaga-se por toda sua extensão, apesar do fato de que as interações entre unidades são locais. De fato, uma tal perturbação decai, mas de forma algébrica, em vez da forma exponencial observada para os valores não críticos do parâmetro. Devido a este fato, os aglomerados de unidades correlacionadas seguem uma distribuição de tamanhos que é uma lei de potências. Para modelos exibindo CAO, observam-se também leis de potências para vários fenômenos de interesse. Entretanto, neste caso, o sistema se encontra por si só no estado crítico, sem que haja necessidade de sintonizações por um parâmetro externo.

Por outro lado, é possível estabelecer uma conexão entre sistemas ordinários que passam por uma transição de fase contínua para um estado absorvente e sistemas que exibem CAO. Segundo Dickman, Muñoz, Vespignani e Zapperi [5], sistemas que estão sujeitos a uma lei de conservação e exibem transição de fase entre um estado ativo e um dentre muitos estados absorventes podem ser transformados em sistemas que exibem $\mathrm{CAO}$, introduzindo-se regras que efetivamente forçam o sistema a caminhar para o estado crítico e permanecer lá. Tais regras são um processo de dissipação sempre que o sistema se encontra no estado ativo, a uma taxa muito menor que as da dinâmica original, e um processo de adição a uma taxa muito menor que a de dissipação.

Assim, descobrimos um ponto de partida para a observação de CAO, composto por sistemas que possuem estados absorventes para uma faixa de valores do parâmetro de controle, o qual deve ser uma grandeza conservada pela dinâmica do sistema.

\subsection{Modelos com infinitos estados absorventes}

Dentre os modelos úteis como ponto de partida para se observar CAO, destacamse os modelos de exclusão (não se permite que duas ou mais partículas ocupem o mesmo sítio), com conservação de partículas e a presença de infinitos estados absorventes. Vamos estudar alguns destes modelos, os quais são parcialmente inspirados no processo de contato conservativo introduzido por Tomé e Oliveira [6]. Nesse processo, partículas saltam de um sítio a outro de uma rede, podendo cair apenas em sítios vazios vizinhos de um sítio ocupado, sendo proibidas as quedas sobre sítios vazios isolados. Estudos sobre este modelo mostram que, no limite termodinâmico, suas propriedades são idênticas às do processo de contato conven- 
cional (não-conservativo), de forma que podemos identificar os dois processos como realizações de um mesmo sistema em ensembles estatísticos diferentes.

O processo de contato convencional possui apenas um estado absorvente, caindo na classe de universalidade da percolação direcionada, a qual pode ser caracterizada pelo expoente crítico $\beta=0.2765$. Devido à equivalência de ensembles no limite termodinâmico, podemos dizer que o processo de contato conservativo também cai nessa classe de universalidade.

Entretanto, podemos encontrar outras classes de universalidade, partindo de modelos que possuem um número infinito (no limite termodinâmico) de estados absorventes. Tais modelos incluem caminhantes aleatórios ativados [5] e o processo de contato conservativo invertido, definido no capítulo 2 .

Neste trabalho, vamos estudar modelos com regras de transição que permitem que uma partícula salte de um sítio para outro se o sítio original for vizinho de um sítio ocupado e o sítio final estiver vazio, além de outras regras suplementares. Se o sítio de destino pudesse estar localizado em qualquer posição da rede, as transições permitidas pela primeira regra seriam as inversas das transições permitidas no processo de contato conservativo. Entretanto, impomos uma restrição quanto ao sítio de chegada em todos os modelos estudados, o qual deve estar a $n$ sítios de distância em relação ao sítio de partida, de modo que sítios distantes não influem nas taxas de transição.

A existência das regras suplementares garante que o estado estacionário ativo possa ser descrito como um conjunto de configurações onde os sítios vazios agrupamse em aglomerados de tamanho definido (contendo 1 ou 2 partículas), num mar de sítios ocupados, enquanto continuam existindo infinitos estados absorventes. É possível mostrar que tais estados são ergódicos para alguns modelos, ou seja, é possível alcançar qualquer configuração permitida partindo de uma configuração qualquer. Quando esta condição é satisfeita, podemos utilizar o conceito de ensembles estatísticos desenvolvido por Gibbs para calcular as grandezas físicas necessárias, efetuando uma mudança de ensemble que nos permitirá utilizar a técnica de matrizes de transferência. O cálculo do maior autovalor desta matriz em função da atividade $z$ nos leva a uma expressão para o cálculo das diversas grandezas físicas.

Este trabalho está organizado como se segue: nos capítulos 2 e 3, apresentamos algumas considerações teóricas necessárias sobre processos estocásticos em reticulados, ensembles estatísticos e matrizes de transferência necessários ao acompanhamento dos demais capítulos. Os capítulos 4, 5, 6 e 7 apresentam os modelos estuda- 
dos detalhadamente neste trabalho, que podem ser descritos através da técnica de matrizes de transferência, enquanto o capítulo 8 apresenta contra-exemplos do uso desta técnica. Discussões finais e conclusões são feitas no capítulo 9. 